\title{
Preparation of radiological inputs for environmental impact assessment of routine normal operation of nuclear facilities
}

\author{
P. Pecha and E. Pechova ${ }^{1}$
}

\author{
Institute of Information Theory and Automation, Academy of Sciences of the Czech Republic, \\ Pod Vodarenskou Vezi 4, 18208 Prague 8, Czech Republic \\ ${ }^{1}$ ENERGOPROJEKT Prague, Vyskocilova 3/741, 14021 Prague 4, Czech Republic
}

\begin{abstract}
The paper describes the input data generation for EIA (Environmental Impact Assessment) procedure based on the latest US EPA methodology for case of radioactive discharges from nuclear facilities to the living environment. Previous concept of numerical dose factors (or dose coefficient) for estimation of radiation doses due to exposure to radionuclides is in the EPA model extended to the numerical risk coefficients which enable alternative estimation of risk to health from exposure to radionuclides. The main characteristics of radiological situation around the source of pollution are computed on the basis of profound analysis of radionuclides propagation in atmosphere, hydrosphere and their further food-chain transport. The risk assessment is then done such a product of the risk coefficients and the main characteristics. A special software tool was developed and is presented here enabling to distinguish between short-term acute exposure scenarios and chronic long-term processes resulting in constant concentration (e. g. annually averaged) of a radionuclide in a given environmental medium. Particular attention was dedicated to ingestion pathway where dynamic models for computation of the annual activity intakes were customised to the local Czech conditions. Two cases of one-time radioactive fallout in a certain Julian day of a year (accidental releases) and long-term continuous submersion of ecosystem into the contaminated environment (normal releases during routine nuclear power plant (NPP) operation) are described by two different ingestion algorithms.
\end{abstract}

\section{ASSESSMENT OF ENVIRONMENTAL EXPOSURE TO RADIONUCLIDES}

Health consequences of radioactive releases into the living environment cover wide range of deterministic and stochastic effects. The principal stochastic somatic health effects are the increased incidence of cancers in the irradiated population. One of the most important issue resulting from the EIA (Environmental Impact Assessment) procedure is the cancer risk assessment. In a common practice the estimation of the cancer risk from activity intake and external exposure to emitted radiation is done according to ICRP 60 recommendation such a simple product of an estimated effective dose and so called nominal probability coefficients in $\mathrm{Sv}^{-1}$. The nominal values do not account for the inherent uncertainties and are based on the idealised adult population receiving a uniform dose over the whole body. Some other problems of the approach are pointed out by the ICRP itself.

An alternative approach to the environmental impact assessment concentrated on the stochastic cancer risk of radioactive discharges is based on the latest US EPA (United States Environmental Protection Agency) methodology [1]. It provides the new sets of risk coefficients which take into account the age dependence of biological behaviour and then characterise more precisely the implications of age and gender dependencies of activity intake, metabolism, radiogenic risk and competing causes of death. For a given radionuclide and exposure mode the corresponding mortality risk coefficient estimates the risk of dying from cancer for an average person. The coefficients are related to the unit activity inhaled or ingested for internal exposure and to the unit time-integrated activity concentration in air or soil for external exposure. From the point of view of different scenarios, a risk coefficient $\boldsymbol{r}$ may be interpreted as a risk per unit exposure for a typical person exposed throughout life to a constant concentration o: radionuclide in an environmental medium (chronic exposure), or as the average risk per unit exposure tc members of a stationary population that experiences an acute exposure to the radionuclide in a certai environmental medium. 
The EPA methodology provides the risk coefficients estimating the risk of cancer from low-level exposure of radionuclides and presumes that the risk is directly proportional to intake or exposure according the linear no-threshold model (LNT). For a given exposure scenario the overall lifetime cancer risk $R$ evoked by the driving quantities $X, Y$ and activity intake $I(X: Y)$ are expressed as $R=r_{e x} . X$ for external exposure and $R=r_{i n}, I(X i Y)$ for internal exposure . Then, for the assessment based on the US EPA methodology it is necessary to find detailed spatial activity distribution of the main driving variables $X, Y$ and $I(X, Y)$ around the source of pollution. Two driving variables $X$ express the time integrated activity concentrations of nuclide $\underline{n}$, specifically $\boldsymbol{T I C}^{n}{ }_{\text {air }}$ in the near-ground layer of the air [Bq.s. $\left.\mathrm{m}^{-3}\right]$ and $T I C^{n}{ }_{g r}$ on the ground surface [Bq.s.m ${ }^{-2}$ ]. Two main variables $Y$ express either specific activity deposition on the ground $\Omega_{g r}^{n}$ just after the plume passage over the terrain [Bq. $\mathrm{m}^{-2}$ ] for acute scenario or annually averaged long term value of specific activity deposition rate Drate $_{{ }_{g r}} \quad\left[\mathrm{~Bq} \cdot \mathrm{m}^{-2} \cdot \mathrm{s}^{-1}\right]$. Specifically, for the internal activity intake is used the notation:

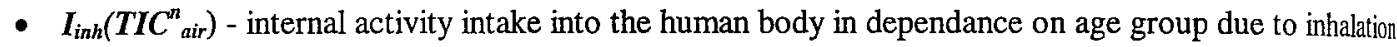
(in Bq per integration time)

- $I_{\text {ing }}\left(\Omega^{n}{ }_{g r}\right), I_{\text {ing }}\left(\right.$ Drate $\left._{g r}\right)$ - internal activity intake into due to ingestion of contaminated food either for acute or chronic scenarios (in $\mathrm{Bq}$ per duration of consumption); in dependence on age group and the Julian day of fallout (acute)

The current distribution of the risk probabilities around a certain nuclear facility is then defined as a product of the risk coefficients from [1] and the corresponding values of spatial activity distribution generated by program codes HAVAR [6] and NORMAL [4] for cases of the accidental or routine normal operation scenarios. The generation procedure is briefly mentioned in the following text.

\section{TRANSPORT OF RADIOACTIVE DISCHARGES IN ATMOSPHERE}

Ones the radioactive material is released from venting stacks, containment or building roofs, the admixtures are incorporated in the plume and drifted in the downwind direction. The polluted plume expands horizontally and vertically due to turbulent diffusion in the atmosphere. The radionuclides are bound in the plume in a certain physical-chemical forms (aerosols, elemental form, organically bound) and during the dispersion are removed from the plume due to several removal mechanisms. The process results in the plume depletion, mainly due to:

- radioactive decay - daughter build-up have to be taken into account for activity in air during the plume travel and for deposited activity during the period of its persistence on the ground

- dry deposition - gravitational setting and deposition due to contact of the contaminated plume with the ground, vegetation or urban structures

- wet deposition - removal by rainout (precipitation formation process inside of the plume) or by washout (interaction between falling drops and admixtures)

Atmospheric dispersion of airborne nuclides is determined by mechanisms of advection and diffusion transport. The advection model provides wind field characteristics. The diffusion processes in the planetary boundary layer are initiated by turbulent motion of atmosphere and cause the spread of admixtures vertically and peripherally to the transport direction. Segmented Gaussian straight-line diffusion model is used here. Diffusion categories according to Pasquill-Gifford notation enable to distinguish various turbulent and thermal states of atmosphere.

Within wind field and dispersion calculations many other factors have to be taken into account such thermal structure of the atmosphere, surface roughness and other land cover characteristics, orography of the terrain, reflection from the ground and top of the mixing layer or inversion layers, the effect of initial plume rise due to vertical momentum and buoyancy, recirculation in the wake region of the near standing buildings. Diffusion parameters $\sigma_{\mathrm{y}}(\mathrm{x})$ and $\sigma_{\mathrm{z}}(\mathrm{x})$ and their dependence on downwind direction $\underline{x}$, stability category, surface roughness and effective release height are taken into account for both urban and rural areas. These effects are usually expressed by semi-empirical formulas derived from experimental results. 
Nevertheless it includes a certain degree of uncertainties. That is why the several alternative models are available in NORMAL or HAVAR products which enable user to analyse the problem from different pints of view. A special attention has been dedicated in [9] to the categorisation of land use types with respect to its local effect on dry deposition velocity and surface roughness which led to an improvement of source depletion model used within the Gaussian solution.

The dispersion problem is solved on the polar grid with 16 angular sectors of windrose and 35 radial distances up to $100 \mathrm{~km}$ from the source. Let us consider one real situation with category of atmospheric stability $\dot{L}$, when wind blows in direction of $\underline{k}$ of windrose with speed from wind category $\underline{m}$. Moreover, atmospheric precipitation occurs with intensity from category $\underline{s}$, inversion situation occurs with inversion height from category $g(s=1, g=1 \Rightarrow$ no precipitation, no inversion occur $)$. The spatial and time $(t)$ distribution of the activity concentration $\mathrm{C}_{\mathrm{k}}{ }^{\mathrm{n}}(\mathrm{x}, \mathrm{y}, \mathrm{z}, \mathrm{t} ; \mathrm{j}, \mathrm{m}, \mathrm{s}, \mathrm{g})$ of radionuclide $\underline{n}$ in air is given by the stepwise Gaussian straight-line solution. Coordinates $\underline{x}, \underline{y}$ and $z$ denote downwind, peripheral and vertical directions. Corresponding near-ground variable $\mathrm{C}_{\mathrm{k}}{ }^{\mathrm{n}}(\mathrm{x}, \mathrm{z}=0, \mathrm{t} ; \mathrm{j}, \mathrm{m}, \mathrm{s}, \mathrm{g})$ is interpreted as maximum under the plume axis (in windrose direction $\underline{k}$ ) for accidental scenarios and as averaged within the angular sector $\underline{k}$ for normal routine releases.

For case of long-term normal operation with routine releases the calculations are repeated for all possible values of the indexes $j, m, s, g$ and the resulting mean anmual values are computed by weighting the particular values by annual weather statistics $\mathrm{QW}(\mathrm{k}, \mathrm{j}, \mathrm{m}, \mathrm{s}, \mathrm{g})$ given by the National Meteorological Service. The final annual mean activity concentration $\mathrm{MC}_{\mathrm{k}}{ }^{n}(\mathrm{x}, \mathrm{Z}=0)$ of radionuclide $\underline{n}$ in the direction $\underline{k}$ (averaged within sector $\underline{k}$, e.g. in peripheral dir.) and radial distance $\underline{x}$ from the source is schematically expressed as:

$$
M C_{k}^{n}(x, z=0)=\sum_{(j)} \sum_{(m)} \sum_{(s)} \sum_{(g)} C_{k}^{n}(x, z=0 ; j, m, s, g) \cdot \mathrm{QW}(\mathrm{k}, \mathrm{j}, \mathrm{m}, \mathrm{s}, \mathrm{g})
$$

\section{DEPOSITION OF ACTIVITY ON THE GROUND}

Due to dry and wet removal processes of admixtures from the plume the radionuclides are deposited on the ground. Time dependency of deposited activity $\Omega^{n}{ }_{g r}(t ; x, y)$ is found from the activity balance equation where the radioactive decay and removal of the nuclide from the surface (e.g. migration, run-off mechanisms) mean negative terms and contribution from the parent nuclides and from the activity deposition rate from fallout and washout represent the sources. The key value of the mean deposition rate $\left[B q . \mathrm{m}^{-2} \cdot \mathrm{s}^{-1}\right]$ for case of normal long-term routine releases Drate $_{\mathrm{gr}}^{n}$ is now equivalent to the source term:

$$
\bar{D} \text { rate }_{g r}^{n}(t ; x)=\dot{A}^{n} \cdot\left[\bar{F}_{k}^{n}(t ; x)+\bar{W}_{k}^{n}(t ; x)\right]
$$

where $\dot{A}^{n}, \bar{F}_{k}^{n}(t ; x), \bar{W}_{k}^{n}(t ; x)$ denote release rate of nuclide $\underline{n}$ from the source [Bq. $\left.\mathrm{s}^{-1}\right]$ (homogenized during year) and long-term (annually averaged, mean within sector $\underline{k}$ ) fallout and washout coefficients (both are calculated similarly to eq. (1) - details in [4]). The scheme (2) with peripheral dependency $y$ holds true also for the case of accidental releases described by segmented Gaussian plume model, where within one segment the release rate $A^{n}$ is assumed to be constant and the quantities $F$ and $W$ are substituted by their short-term values [6]. Detail knowledge of the deposition rate enables further calculation of activity depletion from the plume and detailed time dependency of the activity deposited on the ground both for chronic and acute scenarios. Such an example is given on fig. 1 the 2-D plot of annual nean values of deposited activity of radionuclide Cs137 on the ground. Prevalent annual wind directions resulted from the annual weather statistics can be recognized from here. Two isolines (4e-05 - violet color originally; 2e-05 - yellow orig.; in [Bq. $\left.\mathrm{m}^{-2}\right]$ ) of the annual values of deposited activity of Cs 137 are related to the total annual release of $\mathrm{Cs} 137=5.97 \mathrm{e}+5 \mathrm{~Bq}$ /year (from obligatory validation task no. 2 defined by the Czech Accreditation Board for the codes used in the field nuclear safety including national code 
NORMAL). The results are displayed over a real map background of the locality of NPP Dukovany. The picture was generated on the screen by the interactive presentation module of the system NORMAL.

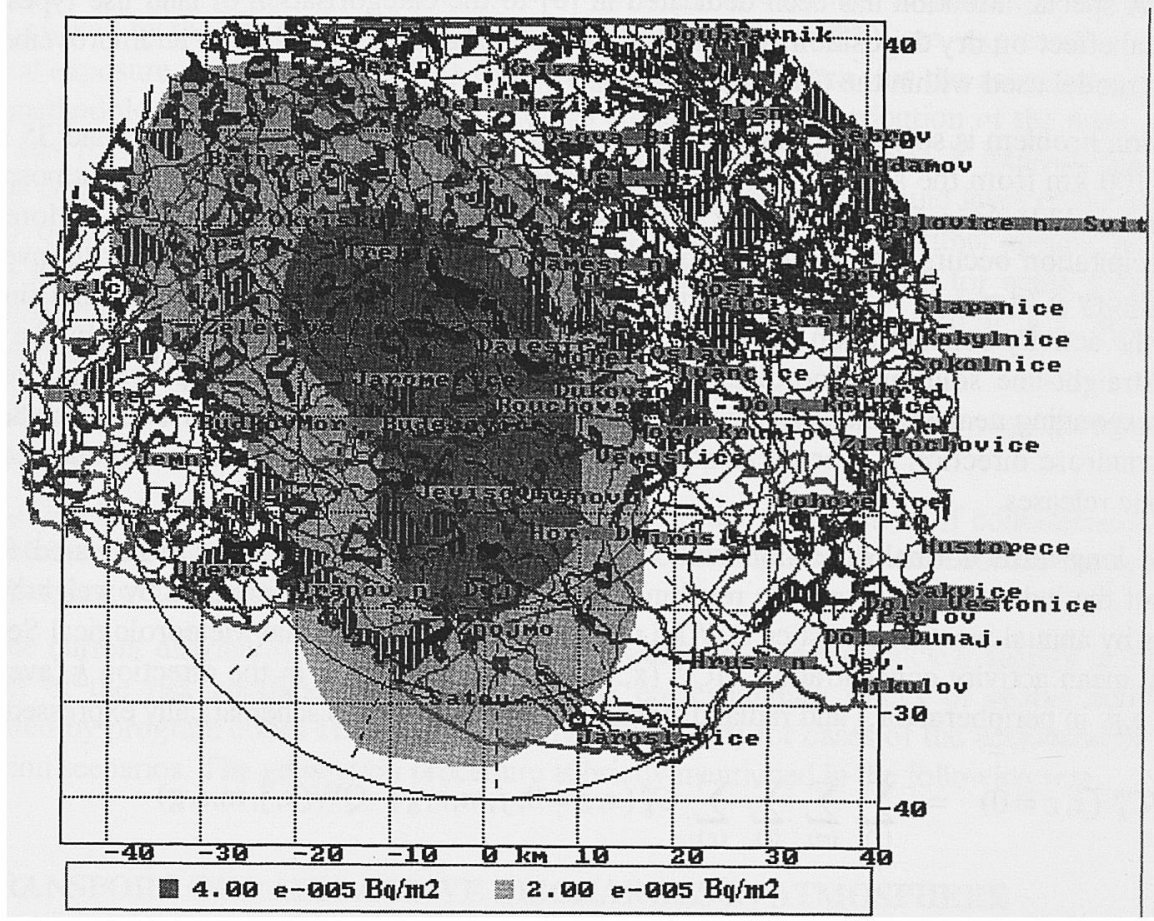

Fig. 1: Annual specific activity of Cs137 deposited on the ground around NPP Dukovany (after the Ist yeat of normal NPP operation, total year release of Cs137=5.97 E+5 Butyeat)

\section{INPUTS FOR THE INTERNAL EXPOSURE ASSESSMENT DUE TO ACTIVITY INHALED AND INGESTED}

\subsection{Internal intake of activity due to inhalation pathway}

The inhaled activity $\boldsymbol{I}_{\text {inh }}\left(\boldsymbol{T I C ^ { n }}{ }_{\text {air }}\right)$ of nuclide $\underline{n}$ during a certain time $\mathrm{T}$ is computed as a product of breathing rate and the value of integrated (during the T period) near-ground activity concentration of $\underline{n}$ in air. The breathing rates have to be distinguished according to age groups. The $\boldsymbol{T I C}_{\text {air }}{ }$ values are generated in dependency on the release scenario as:

- For accidental (acute) scenarios - on the basis of time integration of variable $\mathrm{C}_{\mathrm{k}}{ }^{\mathrm{n}}(\mathrm{x}, \mathrm{y}, \mathrm{z}=0, \mathrm{t} ; \mathrm{j}, \mathrm{m}, \mathrm{s}, \mathrm{g})$

- For chronic scenarios (e.g. routine releases during normal NPP operation) - the annually averaged values of $\mathrm{MC}_{\mathrm{k}}{ }^{\mathrm{n}}(\mathrm{x}, \mathrm{z}=0)$ from eq. (1) are multiplied by the selected time interval $\mathrm{T}$.

\subsection{Internal intake of activity due to ingestion pathway}

The food chain transport of radionuclides is treated again in relation to the exposure scenarios. Two different approaches have been developed for:

- Short-term accidental releases - the acute exposure due to internal activity intake caused by consumption of contaminated foodstuffs $I_{i n g}\left(\Omega^{n}\right)$ is estimated on the basis of initial spatial distribution of activity deposited on the ground just after the radioactive plume pass the terrain in a certain Julian day of fallout in a year. The corresponding algorithm described in [6] come out from the model ENCONAN [7] (simplified ECOSYS methodology) where the first attempt to adjust it for the Czech local conditions is done. Sensitivity of the annual activity intake dependence on Julian day of radioactive fallout with regard to vegetation periods of agricultural products was analysed in [6]. 
- Chronic long-term releases - During normal NPP operation the ecosystem surrounding the NPP is assumed to be continuously submerged into the contaminated environment where customary agricultural practices are applied. Unlike to the short-term release the detailed modelling of continuous activity deposition process comes out from the activity deposition rate distribution expressed by eq. (2). In addition, the transport of tritium and carbon-14 is assumed to be driven by the photosynthesis process and then dependent on the $\mathrm{MC}_{\mathrm{k}}{ }^{\mathrm{n}}$ values of concentration from eq. (1).

The new food-chain transport model for the latter case of long-term releases is introduced in $[4,5]$ and enables more precise generation of annual activity intake. The dynamic model is adopted for the average Czech conditions taking into account local consumption habits (dependence on season and age), agricultural production scheme, average agro-climatic conditions and phenologic characteristics of the plants, feeding diets of animals, time delays during processing, transport and storage of foodstuffs and feedstuffs etc. The balance equation of the activity continuously deposited on the leaves and ground assumes source term expressed by the deposition rate according to (2) and two different mechanisms of nuclides uptake into edible parts of plants are modelled:

Foliar uptake of radionuclides: In the process of the continuous deposition on leaves the initial deposited activity is decreased due to weathering effects (wind, rain), radioactive decay and tissue ageing (growth dilution effect). Furthermore, the fraction of activity translocated to other parts of the plant should be taken into account. The analysis must distinguish between plants which are used totally (e.g. leafy vegetables, grass) and plant of which only a special part is used (cereals, potatoes). Translocation from leaves to the edible parts of the plant has to be accepted. This process is strongly dependent on the physiological behaviour of the isotope considered.

Root uptake of radionuclides: In general, the root uptake of activity is calculated from the concentration of activity in the soil using equilibrium transfer factors which give the ratio of activity concentration in plants (fresh or dry weight) to soil (dry soil). Cumulation of ground activity during years is accepted.

Total time integrated intake of activity (during time period $\underline{t}$, normalised to the unit deposition rate of nuclide $\underline{n}$ ) for age category $\underline{a}$ due to both direct consumption of edible parts of plants and consumption of contaminated animal products is schematically written as

$$
I \mathcal{E}_{\text {TOT }}^{a, n}(t)=\sum_{(l)} I \mathcal{E}_{l}^{a, n}(t)+\sum_{(b)}\left\{\sum_{p, b} I \mathcal{E}^{a, n}(t)\right\}
$$

Here $\underline{l}$ denotes the plant products, $\underline{b}$ means the animal products (milk, meat, eggs). From the products $\underline{b}$ are produced various foodstuffs $\underline{p}(\underline{b})$ (for example from the milk are produced the foodstuffs $\underline{p}$ : fresh milk, cream, cheese, milk dry, milk condensed, curd, others - with various specific time delays for consumption). The Czech local age-dependent consumption basket and local dynamic parameters are implicitly included. For $t=1$ year the terms on the right side of (3) represent the total annual activity intake (normalised to the unit deposition rate) from the direct consumption and from consumption of animal products. Resulting intake $\boldsymbol{I}_{\text {ing }}\left(\right.$ Drate $\left._{{ }_{g r}}\right)$ is found by multiplying the values from (3) by real distribution of Drate $^{n}{ }_{g r}$. In the following table 1 are included results for adult category for several isotopes from a given spectrum of annual releases of nuclides predicted for routine operation of NPP Temelin.

Tab. 1 : Normalised annual activity intake of isotopes due to ingestion pathway - adults

(related to the local Czech dynamic parameters and consumption basket)

\begin{tabular}{|c|c|c|c|c|c|c|c|c|}
\hline \multirow{2}{*}{$\begin{array}{l}\text { Year after start of } \\
\text { NPP operation }\end{array}$} & \multicolumn{8}{|c|}{ radionuclide } \\
\hline & $\mathbf{H 3}^{\left({ }^{*}\right)}$ & $\mathrm{C14}^{(*)}$ & Co60 & Ni63 & $\mathbf{I 1 3 1}$ & Cs134 & Cs137 & $\cdots \cdots$ \\
\hline in the $1^{\text {st }}$ year & $0.12 \mathrm{E}+5$ & $0.22 \mathrm{E}+6$ & $0.19 \mathrm{E}+9$ & $0.18 \mathrm{E}+9$ & $0.51 \mathrm{E}+7$ & $0.25 \mathrm{E}+9$ & $0.29 E+9$ & $\cdots$ \\
\hline in the $31^{\text {st }}$ year & $0.12 \mathrm{E}+5$ & $0.22 \mathrm{E}+6$ & $0.26 \mathrm{E}+9$ & $0.62 \mathrm{E}+9$ & $0.51 \mathrm{E}+7$ & $0.27 \mathrm{E}+9$ & $0.36 \mathrm{E}+9$ & $\cdots$ \\
\hline
\end{tabular}

"... normalised to unit near-ground act, concentr, in air $\left[\mathrm{m}^{3}\right]$; other nuclides: normalised to unit deposition rate [ $\left.\mathrm{m}^{2} . \mathrm{s}\right]$ 


\section{CONCLUDING REMARKS}

Extensive comparative studies were done with the COSYMA [6] and PC CREAM [4] results (partially with a certain RODOS runs) for the main driving variables $\boldsymbol{X}, \boldsymbol{Y}, \boldsymbol{I}(\boldsymbol{X}, \boldsymbol{Y})$. Furthermore, the flexible new ingestion algorithm was used for analysis of other important effects, such as:

- Estimation of various feeding practices: A certain hypothetical scenario for the 3 cuts of forage and corresponding feeding rates for dairy cows and cattle in fattening was introduced. The combination of indoor fattening in a stable and continuous pasture of the fresh grass can be studied.

- Effects of seasonal consumption: More realistic seasonal scenarios can be treated in consumption scheme of a certain products. Considerable differences were found for some nuclides (e.g. I131) in relation with the average annual consumption.

- With small modification the new ingestion algorithm can be adopted for the estimation of irrigation of plants by contaminated water where the discrete watering is substituted by the equivalent continuous spraying during the whole vegetation period [4]

As for the future prospects of development the three main topics seem to be in the centre of interest when proceed from estimation of conservative potential exposure assessment to the more realistic expected magnitudes:

- Adoption of more realistic ingestion scheme instead of the "local production-local consumption" one. The equilibrium transfer factor concept should be substituted by realistic compartment metabolic models.

- Introduction of multi-regional concept, when the whole territory is split into a certain number of so called radioecological zones according to differences in climate, phenological characteristics, agricultural production and feeding regimes, consumption habits, soil types (strongly influencing soil to plans transfer factors) etc. The first attempt insist in the utilisation of multi-criterion concept for the new regionalization of the Czech Republic from the point of view of global criterion of "favourable vegetation conditions" [10].

- Model parameters improvement, which includes data actualisation and reconstruction of various items on fine spatial grid (site specific data, soil type, land use, elevations, agricultural production, population, more detailed meteorological statistics etc.) related to the local-specific conditions.

\section{References}

1. K. F. Eckerman, R. W. Leggett, C. B. Nelson at al., ORNL EPA 402-R-99-001, FGR No. 13 (1999)

2. J. R. Simmonds, G. Lawson, A. Mayall, in EUR 15760 EN - PC CREAM code, (1995).

3. J. Brown, J. A. Jones, J. Ehrhardt at al., in EUR 16240 EN (NRPB-SR280) - PC COSYMA code (1995).

4. E. Pechova, P. Pecha, NORMAL code - in Tech. rep. EGP Prague, EGP 4104-6-980030 (1999).

5. P. Pecha, E. Pechova, in Proceedings of the $4^{\text {th }}$ Internal. Conf. on Environmental Impact Assessment - EIA - PRAGUE 2000, 11.-14. Sept. 2000, Prague, p. 146.

6. P. Pecha, E. Pechova, HAVAR code - in Tech. rep. EGP Prague, EGP 4104-6-990010 (1999).

7. V. Kliment, Nuclear Energy 38, No. 3, p. 177 (1992).

8. P. Pecha, P. Nedoma, E. Pechova, in Proceedings of the 6-th Int. Conf. on Harmonisation within Atmospheric Dispersion Modelling for Regulatory Purposes. Rouen, 1999, p.207.

9. P. Pecha, P. Kuca, E. Pechova, in Proceedings of the 7-th Int. Conf. on Harmonisation within Atmospheric Dispersion Modelling for Regulatory Purposes. Belgirate, 2001, p.489.

10. D. Moravec, J, Votypka, in Climatic Regionalisation of the Czech Republic. Charles Univ. Publishing, Prague, 1998, ISBN 80-7184-417-9.

11. P. Pecha, P. Kuca, P. Nedoma at al., in FDMT Customisation for the Czech Republic. RODOS(WG3)-TN(98)-14 - final version 1999. 